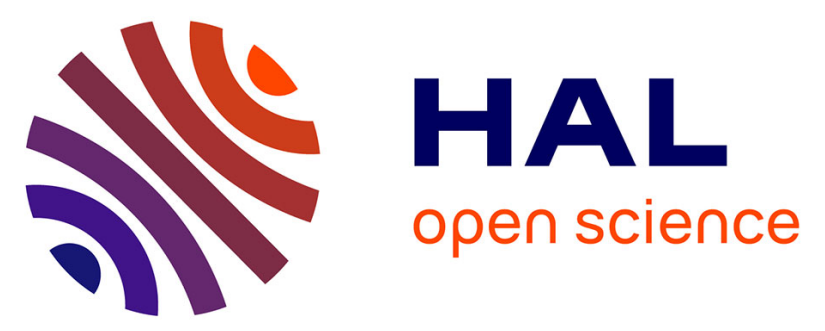

\title{
InGaN Quantum Dots Studied by Correlative Microscopy Techniques for Enhanced Light-Emitting Diodes
}

Ioanna Dimkou, Enrico Di Russo, Pradip Dalapati, Jonathan Houard, Nevine Rochat, David Cooper, Edith Bellet-Amarlic, Adeline Grenier, Eva Monroy, Lorenzo Rigutti

\section{To cite this version:}

Ioanna Dimkou, Enrico Di Russo, Pradip Dalapati, Jonathan Houard, Nevine Rochat, et al.. InGaN Quantum Dots Studied by Correlative Microscopy Techniques for Enhanced Light-Emitting Diodes. ACS Applied Nano Materials, 2020, 3 (10), pp.10133-10143. 10.1021/acsanm.0c02106 hal-02969122

\section{HAL Id: hal-02969122}

\section{https://hal.science/hal-02969122}

Submitted on 16 Oct 2020

HAL is a multi-disciplinary open access archive for the deposit and dissemination of scientific research documents, whether they are published or not. The documents may come from teaching and research institutions in France or abroad, or from public or private research centers.
L'archive ouverte pluridisciplinaire HAL, est destinée au dépôt et à la diffusion de documents scientifiques de niveau recherche, publiés ou non, émanant des établissements d'enseignement et de recherche français ou étrangers, des laboratoires publics ou privés. 


\title{
InGaN Quantum Dots Studied by Correlative Microscopy Techniques for Enhanced Light-Emitting Diodes
}

Ioanna Dimkou, ${ }^{* \dagger}$ Enrico Di Russo, ${ }^{\dagger, \ddagger}$ Pradip Dalapati, ${ }^{\ddagger}, \perp$ Jonathan Houard, ${ }^{\ddagger}$ Nevine Rochat,${ }^{\dagger}$ David Cooper, ${ }^{\dagger}$ Edith Bellet-Amarlic, ${ }^{\S}$ Adeline Grenier, ${ }^{\dagger}$ Eva Monroy, ${ }^{\S}$ and Lorenzo Rigutti

${ }^{\dagger}$ Univ. Grenoble Alpes, CEA, LETI, F-38000 Grenoble, France

*UNIROUEN, CNRS, Groupe de Physique des Matériaux, Normandie Université, 76000 Rouen, France

${ }^{\S}$ Univ. Grenoble-Alpes, CEA, IRIG-PHELIQS, 38000 Grenoble, France

* Corresponding author: ioanna.dimkou@cea.fr

${ }^{\perp}$ Current affiliation: Research Center for Nano-Devices and Advanced Materials, Nagoya Institute of Technology, Nagoya 466-8555, Japan

OrcID:

Ioanna Dimkou: 0000-0002-6865-3820

Enrico Di Russo: 0000-0003-3829-6567

Pradip Dalapati: 0000-0001-9021-726X

Jonathan Houard: 0000-0001-7244-205X

Névine Rochat: 0000-0003-3574-4424

David Cooper: 0000-0003-3479-4374

Edith Bellet-Amarlic: 0000-0003-2977-1725

Eva Monroy: 0000-0001-5481-3267

Lorenzo Rigutti: 0000-0001-9141-7706

\begin{abstract}
:
InGaN/GaN nanostructures form the active region of III-nitride emitters (light emitting diodes, laser diodes, single photon emitters) in the visible spectral range. In order to understand the optical performance of these nanostructures it is necessary to obtain a direct correlation of alloy distribution and optical features. With this purpose in mind, laser-assisted atom probe tomography (La-APT) is a unique tool to visualize the three-dimensional distribution of chemical species at the nanometer scale. Recent advances in this technique also offer the possibility of recording simultaneously the photoluminescence spectrum of the
\end{abstract}


specimen under investigation. Here, we report the results of a correlative study of a stack of InGaN/GaN quantum dots, combining high-resolution chemical and optical characterization by La-APT and in-situ micro-photoluminescence, compared to ex-situ cathodoluminescence. We demonstrate that the structural information extracted from these techniques allows the precise modelling of these nanostructures, obtaining excellent agreement with the optical measurements. During the La-APT experiment, it was possible to resolve the emission of single quantum dots located in the 9 quantum dot layers closer to the substrate. Single quantum dot lines display a spectral shift during the experiment which is assigned to the relaxation of elastic strain due to material evaporation. Our study conveys an image of the Stranski-Krastanov InGaN/GaN quantum dots that is very different from the GaN/AlN systems. The thickness of the InGaN wetting layer is thicker than the part of the dots that protrudes above the layer, and the indium mole fraction in the wetting layer is lower than that in the dots. This perturbed Stranski-Krastanov growth is explained by the surfactant effect of indium, which favors planar growth.

KEYWORDS: InGaN, quantum dots, correlative microscopy, luminescence, laser-assisted atom probe tomography, cathodoluminescence and photoluminescence. 


\section{INTRODUCTION}

III-nitride semiconductors ( $\mathrm{AlN}, \mathrm{GaN}$ and $\mathrm{InN}$ ) with wurtzite crystalline structure [figure 1(a)] are the keystone of blue and green optoelectronics. III-nitride ternary compounds present direct band gap which can be tuned from the near infrared to the ultraviolet $\mathrm{C}$ region (band gap tunable from $0.69 \mathrm{eV}$ for $\mathrm{InN}$ to $6.2 \mathrm{eV}$ for $\mathrm{AlN})^{1,2}$, with the possibility of fabricating type-I heterostructures [figure 1(b)].3,4 In particular, few-nanometer-thick InGaN/GaN quantum wells (QWs) and quantum dots (QDs) form the active region of IIInitride emitters (light emitting diodes, laser diodes, single photon emitters). ${ }^{5-8}$ The localization of carriers in intentional (QDs) or unintentional [alloy fluctuations at the scale of the exciton Bohr radius $(\approx 2.8 \mathrm{~nm}$ in $\mathrm{GaN})]$ quantum confined systems may play a crucial role in their optical properties, having an impact on emission energies, oscillator strength, and eventually the recombination efficiency of the system. ${ }^{9,10}$ Even in the case of QWs, structural factors like alloy fluctuations, extended defects or strain inhomogeneities can induce a weak three-dimensional (3D) confinement of carriers at the nanometer scale, ${ }^{11}$ which reduces the radiative lifetime, but also broadens the emission spectra and possibly enhances Auger recombination, ${ }^{12,13}$ contributing to the efficiency droop in light emitting diodes. ${ }^{14}$ Although the effect of indium fluctuations on the luminescence properties has been extensively discussed, a key challenge has been to correlate optical and structural properties to reach a full understanding of these 3D phenomena.

A similar situation occurs in the case of intentionally created QD structures, ${ }^{15}$ whose optical signature can only be predicted by getting access to the 3D structure and chemical distribution with a resolution close to the atomic scale. ${ }^{16,17}$ In the case of QDs synthesized following the Stranski-Krastanov growth method, most studies correlate typical optical properties with typical structural properties, since the dispersion of size, shape and composition in the QD ensemble sets a limit to precise modeling. The analysis is easier in 
the case of the GaN/AlN system ${ }^{18}$ since interdiffusion is negligible and the QD facets and wetting layer thickness are well defined. ${ }^{19}$ In contrast, the study of Stranski-Krastanov InGaN/GaN QDs ${ }^{15,20-23}$ presents a major challenge, since little is known about their shape, the thickness of their wetting layer and the distribution of indium in the QD+wetting layer ensemble.

For the study of InGaN nanostructures, transmission electron microscopy (TEM) based techniques were called into question since the exposure to the imaging electron beam can induce alloy clustering. ${ }^{24}$ In this direction, laser-assisted atom probe tomography (La-APT) is a valuable alternative providing $3 \mathrm{D}$ chemical information. ${ }^{25,26}$ Furthermore, the possibility of analyzing the luminescence in situ during the La-APT measurement (the so-called photonic atom probe ${ }^{27}$ or PAP technique) allows linking the emission lines to the atomic layers that are evaporated. ${ }^{28}$ However, quantitative La-APT of III-nitrides faces some particular challenges since the result depends on experimental parameters such as the electric field at the sample surface, the laser pulse energy and the sample temperature. ${ }^{29}$

Here, we investigate the correlation of the spectral emission properties of StranskiKrastanov InGaN/GaN QDs with their structural and chemical features, even at the level of a single QD. The structure of the QDs is defined in detail by combining the chemical information extracted by La-APT, the distribution of QD size and shape measured by TEM and atomic force microscopy (AFM), and the superlattice period extracted from x-ray diffraction (XRD) measurements. The model of the QD structure is validated by the measured optical lines and those predicted by theoretical calculations. The study also reveals that the emission linewidth of the QD ensemble is mostly due to in-plane fluctuations of the indium content in the QDs and the dispersion of the QD diameter. 


\section{EXPERIMENTAL SECTION}

Specimen growth. The sample under study consists of 40 periods of InGaN/GaN QDs deposited on commercial 3.5- $\mu$ m-thick GaN-on-sapphire templates by plasma-assisted molecular beam epitaxy (PAMBE). The QD height was $2.0 \pm 0.2 \mathrm{~nm}$ and the barrier thickness was $\approx 10 \mathrm{~nm}$. The growth process was monitored by Reflection high-energy electron diffraction (RHEED). The substrate temperature was fixed at $597^{\circ} \mathrm{C}$ (calibrated by measuring the In desorption time, as described by S. Valdueza-Felip et al. ${ }^{30}$ ), and the active nitrogen flux was adjusted to provide a growth rate of 0.52 monolayers per second (ML/s) under metal-rich conditions. For the generation of InGaN QDs, the Ga flux was fixed at 30\% of the stoichiometric value, and the In flux was tuned close to the stoichiometry, as described in refs. ${ }^{21,31}$. Therefore, the growth proceeds under metal-rich conditions during the whole process, as schematically described in figure 1(c). The Stranski-Krastanov transition is due to an elastic relaxation process forced by the strong lattice mismatch [see figure 1(d)], in spite of the slightly metal-rich atmosphere and the well-known surfactant effect of In, which promotes planar growth. ${ }^{32}$ The InGaN growth time was $45 \mathrm{~s}$, followed by a growth interruption of $15 \mathrm{~s}$, during which roughening was observed in the RHEED pattern. For the deposition of the GaN barriers, the In shutter was closed and the Ga flux was fixed slightly above the stoichiometric value. Before the growth of the following QD layer, the Ga excess accumulated at the growth front is consumed with active nitrogen during $15 \mathrm{~s}$. The substrate was rapidly cooled down after the deposition of the last QD layer, to enable characterization of the QD shape and density by AFM.

Surface and structural characterization. AFM measurements were performed using a Dimension Icon AFM system operated in the tapping mode using Bruker TESPA-V2 tips.

The periodicity and structural quality was analyzed by XRD in a Rigaku SmartLab 
diffractometer using a 2 bounce $\mathrm{Ge}(220)$ monochromator and a long plate collimator of $0.228^{\circ}$ for the secondary optics.

To study the as-grown structure, a lamella specimen extracted from the sample was observed by scanning TEM (STEM) in an aberration-corrected FEI Titan 80-300 microscope operated at $200 \mathrm{kV}$, with high-angle annular dark field (HAADF) and bright field (BF) detectors. In order to minimize electron beam damage, STEM images were recorded in a sample region far from the area used for the alignment process. In this way, the region of interest was exposed to the electronic beam for less than one minute. This prevents electron beam damaging, which is expected to be significant only after several minutes (e.g. in Bennett et $a .^{33}$ the exposure time is 64 minutes).

In the case of the APT tips, BF-TEM analyses were performed on a FEI TECNAI microscope operated at $200 \mathrm{kV}$. Such TEM measurements are required to extract the APT 3D reconstruction algorithm, namely the shank angle and the initial tip curvature radius. The total exposure time was less than 30 seconds.

Optical spectroscopy. Photoluminescence (PL) measurements under continuous-wave excitation were obtained by pumping with a frequency-doubled solid-state laser $(\lambda=244$ $\mathrm{nm})$, with an optical power of $\approx 100 \mu \mathrm{W}$ focused on a spot with a diameter of $\approx 100 \mu \mathrm{m}$. PL measurements under pulsed excitation used a Nd-YAG laser $(266 \mathrm{~nm}, 2 \mathrm{~ns}$ pulses, repetition rate of $8 \mathrm{kHz}$ ). In both cases, samples were mounted on a cold-finger cryostat, and the PL emission was collected by a Jobin Yvon HR460 monochromator equipped with a UVenhanced charge-coupled device (CCD) camera.

Cathodoluminescence (CL) mapping was performed from room temperature to $4 \mathrm{~K}$ using an Attolight CL microscope. The acceleration voltage was $10 \mathrm{kV}$ and the beam current was $\approx 5 \mathrm{nA}$. The luminescence was collected through an integrated microscope objective 
(NA : 0.7). By scanning the sample, the optical spectra of each pixel are recorded on a CCD camera through a dispersive spectrometer with $400 \mathrm{~mm}$ focal length (grating: 150 grooves/mm blazed at 500nm).

Sample preparation. Needle-shaped tips required for atom probe tomography (APT) and TEM lamella specimens were prepared using both FEI Helios NanoLab 450S and Zeiss Crossbeam550 scanning electron microscope/focused ion beam (SEM/FIB). To extract the regions of interest, the as-grown sample was coated with a 500-nm-thick Pt layer using a GATAN PECS system, and $\mathrm{Ga}^{+}$ions were used for the lift-out and annular milling procedure. The annular milling procedure was performed with an acceleration voltage of 15 $\mathrm{kV}$ in a first stage, and then $2 \mathrm{kV}$ for the final milling and cleaning stages, so that the thickness of the amorphized region is reduced. ${ }^{34}$

Atom Probe Analysis. The samples were analyzed in a homemade laser-assisted wideangle tomographic atom probe coupled with a micro-PL ( $\mu \mathrm{PL})$ bench, also referred to as a photonic atom probe (PAP) as described in ref. ${ }^{27,35}$. Analyses were performed at $80 \mathrm{~K}$ and the evaporation of the specimen is controlled and triggered by means of $\approx 150 \mathrm{fs}$ laser pulses at $260 \mathrm{~nm}$, focused on a spot with a diameter around $1.5 \mu \mathrm{m}$. The repetition frequency of the laser was $300 \mathrm{kHz}$ and the average power was $300 \mu \mathrm{W}$. The system was equipped with an 8cm-diameter multichannel plate/delay line detector (MCP/DLD). The flight length is $\approx 10 \mathrm{~cm}$, corresponding to a field of view of $22^{\circ}$ on the detector and of $35^{\circ}$ on the tip. The detection rate was 0.0005-0.0025 event/pulse.

The $\mu \mathrm{PL}$ spectra were acquired in-situ at $80 \mathrm{~K}$. Excitation was provided by the same pulsed laser emitting at $260 \mathrm{~nm}$, using a repetition frequency of $4 \mathrm{MHz}$ to increase the number of counts in the detector. The incident power was tuned in the $25-1000 \mu \mathrm{W}$ range. The PL signal was collected and analyzed through a grating spectrometer with $320 \mathrm{~mm}$ focal 
length equipped with a liquid nitrogen-cooled CCD camera. The spectral resolution was approximately $0.3 \mathrm{~nm}$.

For precise chemical analysis, we also used a CAMECA FlexTAP system. The APT analysis was performed at $50 \mathrm{~K}$ with a UV femtosecond laser emitting at $341 \mathrm{~nm}$ having a pulse energy of $0.2 \mathrm{~nJ}$, laser spot size $\approx 10 \mu \mathrm{m}$, and repetition rate of $50 \mathrm{kHz}$. The field of view was set to $15^{\circ}$. Data were processed using the TAP3D software and homemade MATLAB codes. The 3D reconstruction was performed using a cone-angle algorithm. The reconstruction parameters (cone-angle $=7^{\circ}$ and tip curvature radius $\left.=39 \mathrm{~nm}\right)$ were extracted from the STEM images of the specimen before performing La-APT.

Modeling. Three-dimensional calculations of the band diagram and electronic levels in the QDs were performed using the commercial Schrödinger-Poisson solver nextnano, ${ }^{36}$ with the material parameters described in ref. ${ }^{37}$. The modelled structure consisted of the 10 periods of InGaN quantum dots, with the dimensions and composition extracted by combination of XRD, AFM, STEM and La-APT data. In each layer, the QDs were defined as a hexagonal truncated pyramid with $\{10-13\}$ facets, connected by a wetting layer. The $3 \mathrm{D}$ strain distribution was calculated by minimization of the elastic energy through the application of periodic boundary conditions along the $\langle 1-100\rangle$ and $\langle 11-20\rangle$ directions. For the calculation of the band profiles, the spontaneous and piezoelectric polarization, as well as the band gap deformation potentials, were taken into account. The energy location of the quantum levels and the electron and hole wavefunctions were calculated using the effective mass approximation. This approximation can result in significant deviations from the experiment when the electron levels are located deep into the conduction band due to the nonparabolicity of III-nitrides, ${ }^{38,39}$ but it provides a good description of band-to-band transitions between near-band-edge levels. ${ }^{37}$ 


\section{RESULTS AND DISCUSSION}

Description of the as-grown specimen. The heterostructure under study is a 40-period InGaN/GaN QD superlattice grown by plasma-assisted molecular beam epitaxy on a commercial 4- $\mu$ m-thick GaN-on-sapphire template. Nominally, the heterostructure consists of $\approx 2$-nm-thick InGaN QD layers separated by 10-nm-thick GaN barriers. An AFM image of the topmost QD layer (uncapped) is presented in figure S1 of the Supporting Information. It shows a high density (mid- $10^{11} \mathrm{~cm}^{-2}$ ) of QDs with a height $=0.9 \pm 0.2 \mathrm{~nm}$. Note that this value corresponds to the QD height above the wetting layer. From such AFM measurements, it is difficult to extract reliable measurements of the QD base diameter since the expected value $\left(10-20 \mathrm{~nm}^{31,40}\right)$ is comparable to the dimension of the AFM tip (see figure S2 and associated discussion in the Supporting Information).

The BF-STEM image of the heterostructure in figure 2(a) shows the 40 periods of InGaN/GaN QDs. HAADF- and high-resolution BF-STEM images of the specimen observed along [11-20] zone axis with higher magnification are presented in figure S3 of the Supporting Information. The wetting layer is not well defined due to the high density of dots with a diameter smaller than the thickness of the lamella specimen, $70 \pm 10 \mathrm{~nm}$, which results in more than one dot being projected in the images. It is nevertheless possible to measure with atomic precision the height of the QD layer (including wetting layer), which is $8 \pm 1$ monolayers (ML), i.e. $2.0 \pm 0.3 \mathrm{~nm}$. The diameter of the QDs, estimated from the variations of contrast in the images, would be in the range of $12-17 \mathrm{~nm}$.

Comparing the results of AFM and TEM, we can extract an estimation of the thickness of the wetting layer $(\approx 1.1 \mathrm{~nm})$. This value should be taken cautiously, since the AFM analysis is performed in an uncapped QD layer, and the capping process can lead to 
morphological changes in the QD structures. In the case of GaN/AlN QDs grown by molecular beam epitaxy (MBE), the geometry remains undisturbed during capping for growth temperatures below $730^{\circ} \mathrm{C}$. At higher temperatures, we observed a reduction of thickness that was more severe in the case of plastic relaxation during the growth of the QD layer, i.e. QDs containing misfit dislocations. ${ }^{41}$ In the case of InGaN/GaN grown by MBE, Figge et al. reported the complete dissolution of their InGaN QD structures when overgrown at $510^{\circ} \mathrm{C} .^{42}$ However, their uncapped QDs contained around $80 \%$ of In and there was evidence of plastic relaxation. Other groups have reported stable QD luminescence for InGaN QDs grown by $\mathrm{MBE}$ and capped at significantly higher temperatures (550$\left.640^{\circ} \mathrm{C}\right),{ }^{31,43,44}$ and no effect of capping was observed in the case of InGaN/GaN QDs grown by metalorganic vapor phase epitaxy when the $\mathrm{GaN}$ barriers are grown at the same temperature as the QDs. ${ }^{45}$ In our case, TEM images show that the relaxation process is purely elastic and the GaN barriers were grown at the same temperature as the InGaN QDs. Therefore, in a first approximation, we have considered that the QD height measured by AFM is also valid for capped QDs. The validity of this statement will be further discussed later.

To get access to the average superlattice period and strain state, we performed an XRD study. The reciprocal space map around the (10-15) x-ray reflection of the GaN/InGaN QD superlattice is reported in figure 2(b). The peak corresponding to the GaN substrate is located at the reciprocal lattice point $Q_{x}=-2 /(a \sqrt{3})=-3.625 \mathrm{~nm}^{-1}$ and $Q_{z}=1 /$ $(5 c)=9.625 \mathrm{~nm}^{-1}$, where $a$ and $c$ are the in-plane and out-of-plane lattice parameters. The multiple peaks corresponding to the periodic heterostructure are aligned along $\mathrm{Q}_{\mathrm{x}}=-$ $3.625 \mathrm{~nm}^{-1}$, which demonstrates a pseudomorphic structure on GaN. Precise values of the superlattice period and lattice parameters were extracted from the $\theta-2 \theta$ x-ray diffractogram recorded around the (0002) reflection of $\mathrm{GaN}$, depicted in figure 2(c). The experimental 
result is compared with a theoretical calculation using the Rigaku GobalFit software. The best fit is obtained assuming an average period of $12.6 \pm 0.1 \mathrm{~nm}$, and modeling the QDs as an InGaN layer with a thickness of $1.6 \mathrm{~nm}$ and an average indium composition around $10 \%$. However, the values of InGaN layer thickness and alloy composition must be taken cautiously. The model assumes a pseudomorphic structure on $\mathrm{GaN}$, with biaxial strain in the InGaN layers. In this situation, the out-of-plane strain is given by the expression $\varepsilon_{z z}=$ $-\left(2 c_{13} / c_{33}\right) \varepsilon_{x x}$, where $\varepsilon_{x x}$ is the in-plane strain and $c_{13}$ and $c_{33}$ are elastic constants (we use here $c_{13}=106 \mathrm{GPa}$ and $c_{33}=398 \mathrm{GPa}$ for $\mathrm{GaN},{ }^{46}$ and $c_{13}=92 \mathrm{GPa}$ and $c_{33}=224 \mathrm{GPa}$ for $\mathrm{InN}^{47}$ with a linear interpolation for the ternary alloy). This approximation is not valid in the case of QDs. ${ }^{48-50}$ Therefore, the fit in figure 2(c) tell us only that the QDs can be approximated by layers with a thickness of $1.6 \mathrm{~nm}$ and an average out-of-plane lattice parameter equivalent to that of $\operatorname{In}_{0.1} \mathrm{Ga}_{0.9} \mathrm{~N}$ fully strained on $\mathrm{GaN}$, without any conclusion about the indium mole fraction in the QDs.

The as-grown specimen was characterized by temperature-dependent CL, as illustrated in figure 2(d). The peak emission wavelength is around $413 \mathrm{~nm}$ at $5 \mathrm{~K}$, and shifts to $422 \mathrm{~nm}$ at $300 \mathrm{~K}$. The evolution of the CL integrated intensity normalized to its low temperature value as a function of the inverse temperature is displayed in figure 2(e). The slight increase of the luminescence from $5 \mathrm{~K}$ to $50 \mathrm{~K}$ can be explained by the improved transfer of carriers from the barriers to the dots when increasing the temperature. The integrated intensity remains constant in the range of 50 to $100 \mathrm{~K}$ and drops sharply for higher temperature due to the activation of nonradiative recombination paths. ${ }^{51}$ The dashed line is a fit that assumes dominance of a monoexponential nonradiative process, so that the variation of the normalized intensity is described by

$$
\frac{I(T)}{I_{0}}=\frac{1}{1+A \exp \left(-E_{a} / k T\right)}
$$


where $I_{0}$ is the integrated intensity at low temperature, $A$ is a fitting parameter, $E_{a}$ is the activation energy of the nonradiative process, and $k T$ is the thermal energy. In the figure, $A=11 \pm 3$ and $E_{a}=67 \pm 5 \mathrm{meV}$, which is comparable to the values reported in the literature. $^{21,31}$ The ratio of the room-temperature and low-temperature CL integrated intensities, which is generally considered as an estimation of the internal quantum efficiency, is $52 \%$. The stability of the luminescence with temperature support our assumption that the QDs were not dissolved during the QD process (see comparison of InGaN/GaN QD and QW luminescence in Gačević, et $\left.a l .^{31}\right)$.

To gain further insight on the origin of the CL linewidth, we have performed crosssection CL mapping of a cleaved edge of the sample, with the result displayed in figure S4 of the Supporting Information. Analyzing the CL spectra at different points along the growth axis and focusing on the emission from the InGaN QDs, we observe that the linewidth is rather constant along the structure (with full width at half-maximum FWHM $\approx 20 \mathrm{~nm}$ ), and comparable to that obtained in the top-view configuration. There is a slight shift of the peak emission along the growth axis, but its value $(\approx 2 \mathrm{~nm})$ is of the order of the spectral resolution of the spectrometer $( \pm 0.8 \mathrm{~nm})$ and significantly smaller than the linewidth. Therefore, the spectral broadness of emission is due to in-plane QD inhomogeneties, rather than to a drift of the growth conditions.

At this point, we face some limitations hampering a proper correlation of optical and structural properties, and thus the achievement of a complete understanding of these 3D objects. AFM and TEM provide information on the QD height and density. In addition, XRD gives precise information on the periodicity of the structure. However, we only have a rough estimate of the lateral dimensions of the dots, and no reliable information on the indium distribution in the structure. 
Characterization of the atom probe tomography (APT) specimen. In order to analyze the InGaN/GaN QDs using La-APT, field emission tip specimens were fabricated by standard focused ion beam (FIB) based lift out followed by annular milling procedures. Further details on the specimen preparation are described in the Experimental Section. The FIB process can cause several kinds of damage: ${ }^{52}$ amorphization of the surface due, ${ }^{53} \mathrm{Ga}$ implantation and redeposition of milled material, and formation of voids and bubbles at the surface, which further agglomerate into metallic nano-blitters. ${ }^{54}$ In the case of InGaN, indium redistribution and evaporation is also possible. ${ }^{55}$ It is hence important to be rigorous in the characterization of the specimens before their introduction in the APT system, if results are to be extrapolated to the as-grown material.

FIB prepared tips were first observed in TEM, as illustrated in figure 3(a). The optical activity of the needle-like samples was then measured in the CL system. Figure 3(b) is a panchromatic CL map of the tip in (a), suggesting that the entire superlattice is optically active. Figure 3(c) displays the integrated CL spectrum at room temperature, which presents an emission line at $364 \mathrm{~nm}$ that originates from the GaN substrate, and a large band peaked at $422 \mathrm{~nm}$ that is assigned to recombination in the QDs. The evolution of the emission spectrum along the tip is shown in figure S5 of the Supporting Information. The peak wavelength and linewidth remain approximately constant along the tip (spectral shift smaller than $2 \mathrm{~nm}$ ). The variation of the CL integrated intensity as a function of temperature is described in figure 3(d). The dashed line is a fit to eq 1 where $A=33 \pm 12$ and $E_{a}=38 \pm 10 \mathrm{meV}$, and the room-temperature to high-temperature integrated intensity rate is around $11 \%$. The energy of the QD emission of the tip shape sample remained the same as the as-grown sample, which confirms that the FIB protocol did not introduce significant loss or rearrangement of indium in the specimen. However, the reduced activation energy and 
increased prefactor $A$ points to a moderate enhancement of nonradiative recombination, due to the higher surface-to-volume ratio.

Tips were then introduced in a photonic atom probe (PAP) system that enables in-situ $\mu \mathrm{PL}$ spectroscopy measurements during the La-APT process, as described in the Experimental Section. In order to determine the best experimental conditions to perform simultaneous $\mu \mathrm{PL}$ and APT characterization, we have first measured in-situ $\mu$-PL as a function of the laser pumping power, as show in in figure 4(a). During these preliminary measurements, we used a repetition rate of $3.8 \mathrm{MHz}$, adjusting the laser power in the range of 25 to $700 \mu \mathrm{W}$. In these spectra, it is possible to identify the narrow emission of the GaN substrate at $358 \mathrm{~nm}$ and a broad band peaked around $411 \mathrm{~nm}$, which is assigned to the InGaN QDs. Plotting their intensity as a function of the laser power [figure 4(b)], the luminescence from GaN scales linearly. On the contrary, the intensity of the QD emission saturates for laser power higher than $300 \mu \mathrm{W}$. Figure 4(c) shows the evolution of peak wavelength of the luminescence from $\mathrm{GaN}$ and $\mathrm{InGaN}$ QDs as a function of the laser power. The FWHM of the emission is indicated as an error bar. The GaN line presents a red shift starting at $300 \mu \mathrm{W}$, with an increase of the FWHM. The behavior at high pumping power points to a local increase of the tip temperature, whose effect is dominant over a potential band-filling phenomenon. The spectral shift of the QD band is not so obvious due to the broad emission, but the FWHM increases clearly for laser power higher than $300 \mu \mathrm{W}$.

In view of these results, we decided to perform the APT experiment with a laser energy corresponding to the $300 \mu \mathrm{W}$ experiment, which is the point where the tip starts warming up due to pumping. However, during the APT experiments coupled with $\mu \mathrm{PL}$ the laser frequency was reduced to $340 \mathrm{kHz}$ to allow a time delay between pulses longer than the expected time of flight of the ions from the specimen during the APT process. ${ }^{56}$ 
The in-situ $\mu \mathrm{PL}$ emission during the PAP analysis of the last $15 \mathrm{InGaN} / \mathrm{GaN}$ QD layers is displayed in figure S6 of the Supporting Information. The PL was analyzed during the evaporation of the last 15 QD layers. As described for the preliminary experiments in figure 4, the peak at $358 \mathrm{~nm}$ is related to the carrier recombination in the $\mathrm{GaN}$ buffer, and the broad band around $411 \mathrm{~nm}$ was assigned to the carrier recombination in the InGaN QDs. Figure 5(a) shows a zoomed view of the emission of the QDs acquired at different stages of the tip evaporation. The legend indicates the number of QD layers that were left in the tip when the PL spectra were recorded. Figure 5(b) shows the 3D APT reconstruction of the indium signal when only 5, 3 and 2 layers remain in the specimen, as an example. In figure 5(a), the initial luminescence peak of the whole tip appears broad, as it results from the overlap of the emission of multiple QDs. The spectra do not display major variations during the evaporation of the 5 topmost QD layers (shown in figure S6). However, during the evaporation of the last 10 layers, the total emission intensity decreases, as expected from the reduction of the tip volume containing multiple QDs. As only a few dots remain in the tip, the spectra present a multi-peak structure due to fluctuations of the size and the indium content of the QDs. ${ }^{17}$ When only 5-2 layers are left, we can identify the signature of single InGaN QDs. The QD signal disappears when there is still one $\mathrm{InGaN}$ layer, which means that most probably that first InGaN layer does not contain any dot. Dashed lines in figure 5(a) are Lorentzian fits that identify the QDs contained in layer 2: a first line (QD1) is located at $422.7 \mathrm{~nm}$ with $\mathrm{FWHM}=1.2 \mathrm{~nm}$, and a second emission line (QD2) peaks at $417.36 \mathrm{~nm}$ with FWHM $=0.87 \mathrm{~nm}$. A third peak (QD3) that disappeared after evaporation of layer 3 was located at $413.2 \mathrm{~nm}$ with $\mathrm{FWHM}=1.3 \mathrm{~nm}$.

The evolution of peak wavelength and FWHM of these QD lines (QD1, QD2, QD3) during the evaporation of the tip is described in the figure 5(c), and figure 5(d) shows the evolution of the $\mu \mathrm{PL}$ line assigned to the $\mathrm{GaN}$ buffer (at $358 \mathrm{~nm}$, shown in figure S6). The 
QD emission lines present a red shift of around $2 \mathrm{~nm}$ during the APT process of the last 9 QD layers. ${ }^{35}$ In contrast, the GaN line presents only a slight red shift of around $0.55 \mathrm{~nm}$. The different behavior of QDs and GaN points out that the shift is not thermally induced but rather strain related. Strain is the result of mechanical stress, and it leads to a change of the semiconductor bandgap (which can be estimated using the band deformation potentials) and the generation of piezoelectric polarization. During the experiment, two sources of mechanical stress act on the QDs, namely the stress resulting from the lattice mismatch between $\mathrm{GaN}$ and InGaN and the stress induced by the electric field during the APT measurement. It is hence important to assess the magnitude of both sources of stress to know if the spectral shift is associated to the material properties or to the experimental conditions.

The stress associated with the lattice mismatch between $\mathrm{GaN}$ and $\mathrm{InGaN}$ results in a deformation of the lattice to minimize the elastic energy accumulated during the growth of the sample, as illustrated in figure S7 of the Supporting Information. In the case of the InGaN QDs, there is an in-plane compressive stress due to the growth of InGaN on top of $\mathrm{GaN}$, and a uniaxial compressive stress along the $c$ crystal axis (growth direction) due to the GaN that is in contact with the QD side facets. The stress along the $c$ axis in the QDs can be calculated as

$$
\sigma_{z z}=2 c_{13} \varepsilon_{x x}+c_{33} \varepsilon_{z z}
$$

where $c_{i j}$ are elastic constants and $\varepsilon_{x x}$ and $\varepsilon_{z z}$ are the in-plane and out-of-plane strain. With the values of strain in figure $\mathrm{S} 7\left(\varepsilon_{x x}=-1.15 \%\right.$ and $\left.\varepsilon_{z z}=0.33 \%\right)$, the stress is estimated at $\sigma_{z z}=-1,16 \mathrm{GPa}$. Note the negative sign, which indicates that the stress is compressive along $c$. In this strain configuration, using the deformation potentials from Vurgaftman and Meyer, ${ }^{57}$ the InGaN band gap increases by $\approx 106 \mathrm{meV}$ with respect to the relaxed value. When evaporating the layers on top of a certain QD, the uniaxial stress imposed by the 
material surrounding the dot decreases, but the in-plane stress due to the GaN underlayer remains. Therefore, evaporation should lead to an expansion of the lattice along $c$ (higher $\left.\varepsilon_{z z}\right)$. Keeping $\varepsilon_{x x}=-1.15 \%$ and releasing the uniaxial stress $\left(\sigma_{z z}=0\right)$ would lead to $\varepsilon_{z z}=0.64 \%$, resulting in an increase of the band gap by only $\approx 74 \mathrm{meV}$ with respect to the relaxed value. In addition to this modification of the band gap, the increase of $\varepsilon_{z z}$ induces an enhancement of the piezoelectric polarization. These two phenomena explain a red shift during the APT process of the order of magnitude observed in figure 5(c).

The GaN PL signal in figure 5(d) stems from the GaN layer below the QD superlattice. The topmost nanometers of this layer are strained by the presence of the InGaN QDs on top. Our Nextnano calculations point out strain that can reach $\varepsilon_{x x}=0.15 \%$ and $\varepsilon_{z z}=-0.26 \%$ $\left(\sigma_{z z}=-0.71 \mathrm{GPa}\right)$ right below an InGaN QD. Using again the deformation potentials in ref. 57 , the strain increases the GaN band gap by $\approx 7.6 \mathrm{meV}$. This strain is fully relaxed when the InGaN material is evaporated, which should lead to a slight red shift of the PL signal, as experimentally observed.

On the other hand, during the APT experiment, a high positive voltage in the range of $V_{D C}=5-10 \mathrm{kV}$ is applied to the InGaN/GaN tip, which results in an electric field $F$ at the tip that can be estimated by ${ }^{58}$

$$
F=\frac{V_{D C}}{k R}
$$

where $k \approx 7$ is a geometrical factor and $R$ is the curvature radius of the tip apex. Free carriers generated by laser illumination drift due to the electric field and holes accumulate at the needle apex screening the electric field inside the semiconductor material. However, the field induces a mechanical stress along the tip, which is given by

$$
\sigma_{F}=\frac{1}{2} \varepsilon_{0} F^{2}
$$


where $\varepsilon_{0}$ is the vacuum permittivity. As we evaporate the sample, $V_{D C}$ increases and the radius of the tip, $R$, increases. Tracing those values, the average electric field when the apex evolves along the 9 deepest QD layers can be estimated to be in the range $8-10 \mathrm{MV} / \mathrm{cm}$, corresponding to a field-induced stress in the range $\sigma_{F} \approx 0.25 \mathrm{GPa}$ to $\sigma_{F} \approx 0.42 \mathrm{GPa}$. As the electric field points towards the detector, the stress is uniaxial, tensile, along the $c$ axis of the semiconductor crystal. The field-related stress $\sigma_{F}$ has the opposite direction of $\sigma_{z z}$; however, both $\sigma_{F}$ and $\sigma_{z z}$ increase during the experiment ( $\sigma_{z z}$ becomes less negative and $\sigma_{F}$ becomes more positive). However, it should be kept in mind that $\sigma_{F}$ is at least 4 times smaller than $\sigma_{z z}$ in the QDs, and about half the value of $\sigma_{z z}$ in the GaN material right below the superlattice. Furthermore, $\sigma_{F}$ is expected to increase only by $10 \%$ for an increase of the tip curvature radius of $5 \%$. Therefore, we can conclude that it is the variation of the stress induced by the lattice mismatch that dominates the spectral shift of the PL during the APT measurement. This is further supported by the fact that, at the beginning of the experiment, there is no major difference in the PL peak energy with and without electric field.

It should be mentioned that the PAP requires the use of a fast APT detection system, at the expense of a lower detection efficiency and accuracy in assessing composition. Therefore, in order to quantify the alloy composition within InGaN layers, La-APT measurements were performed using a CAMECA FlexTAP system, equipped with an advanced delay-line-detector (aDLD). ${ }^{59}$ Figure 5(e) shows the indium site fraction map obtained through the analysis of $18 \mathrm{InGaN}$ QD layers present in a 3D APT reconstruction. The InGaN layers contain In-rich regions with a typical in-plane diameter of $15.4 \pm 1.4 \mathrm{~nm}$ and typical In-site fraction $\approx 13 \%$, connected by the wetting layer, a thinner InGaN layer with an In-site fraction $\approx 6 \%$. The shape and size of these In-rich regions correspond to InGaN QDs rather than to random alloy fluctuations. ${ }^{60}$ This picture is confirmed by the complementary isosurface analysis of one of the QD layers [marked with a square in figure 
5(e)], which is reported in figures 5(f) and 5(g). Figure 5(f) displays the isosurface corresponding to an In-site fraction of $14 \%$ (in red), representing a single QD. The diameter of the QD is $15.0 \pm 0.5 \mathrm{~nm}$, with a height of $2.0 \pm 0.1 \mathrm{~nm}$. On the other hand, the isosurface corresponding to an In-site fraction of $6 \%$ [displayed in figure $5(\mathrm{~g})$ ] shows a layer connecting neighboring QDs, consistent with the wetting layer. The combination of both isosurfaces provides a consistent representation of composition and morphology of both wetting layer and QD.

Modelling of the QD structure. At this point, combining the result of STEM, XRD, AFM and La-APT, we have a complete description of the QD structure, including the period of the superlattice $(12.6 \mathrm{~nm}$, from XRD), the height of the QDs $(2.0 \mathrm{~nm}$, from STEM), the QD height above the wetting layer ( $0.9 \mathrm{~nm}$ from AFM), and the In content of the QDs and of the wetting layer (13\% and 6\%, respectively, from APT). To validate these results, we have compared the optical emission with theoretical calculations of the electronic structure using the geometrical and chemical parameters listed above. A schematic description of the simulated structure is presented in figure 6(a), and a complete description of the modelled cell and conditions are described in the Experimental Section. In a first stage of the 3D simulations, we obtain the strain distribution in the structure. The in-plane and out-of-plane components of the strain are presented in figure S7 of the Supporting Information. In the center of the dot, the $\varepsilon_{z z} / \varepsilon_{x x}$ ratio is -0.284 , which confirms a significant deviation from the biaxial strain configuration, where $\varepsilon_{z z} / \varepsilon_{x x}=-\left(2 c_{13} / c_{33}\right)=-0.556$. Taking into account the In content and the calculation of the elastic strain, the results are consistent with XRD measurements: Assuming that the structure is well described by figure 6(a), and with an $\mathrm{In}_{0.06} \mathrm{Ga}_{0.94} \mathrm{~N}$ wetting layer that is biaxially strained and $\mathrm{In}_{0.13} \mathrm{Ga}_{0.87} \mathrm{~N}$ QDs with $\varepsilon_{z z} / \varepsilon_{x x}=$ -0.284 , the average lattice parameter in a period of the superlattice is approximately the same as in an InGaN/GaN QW superlattice with the same thickness and pseudomorphic, 1.6- 
nm-thick $\operatorname{In}_{0.1} \mathrm{Ga}_{0.9} \mathrm{~N}$ QWs, i.e. the structure used for the XRD calculation presented in figure 2(c). The consistency of the results with the structural characterization data supports our initial assumption that the fact of capping the QDs with GaN does not introduce a significant perturbation of their morphology.

Taking the strain distribution into account, the band diagram of the 3D structure was calculated. The band profile along [0001] axis crossing the center of the QDs is displayed in figures 6(b). The electron and hole quantum confined levels are calculated in a QD in the center of the stack. The electron-hole transition at room temperature is predicted to occur at $426 \mathrm{~nm}$, in agreement with the experimental CL peak wavelength. Figure 6(c) and (d) show the electron and hole probability distribution, respectively. The polarization-induced internal electric field in the dots shifts the electron wavefunction towards the QD apex, whereas the hole wavefunction is located deep into the base of the hexagonal truncated pyramid. However, the separation of electron and hole along the growth axis remains relatively small due to the small quantum dot height.

To gain some insight into the variation of the emission wavelength from one dot to another, we performed calculations changing the QD diameter, In content in the wetting layer, In content in the QD, and the QD height [figures 6(e), (f), (g), and (h), respectively] in ranges that reflect the experimentally revealed structural/chemical fluctuations. Red circles in these figures outline the result of the simulation with the nominal input parameters described in figure 6(a). The emission wavelength is particularly sensitive to fluctuations of the indium content in the dot: adjusting the In content from $12 \%$ to $14 \%$, the emission shifts from 419 to $434 \mathrm{~nm}$ [figure 6(g)]. There is also a high sensitivity to the QD base diameter, with the emission shifting from 423 to $430 \mathrm{~nm}$ when the base diameter increases from 14.0 to $16.8 \mathrm{~nm}$ [figure $6(\mathrm{e})$ ]. Note that this shift is larger than that obtained by changing the QD height by $\pm 1 \mathrm{ML}$ (approximately $\pm 0.25 \mathrm{~nm}$ ), in figure $6(\mathrm{~h})$. Keeping in mind that the PL 
emission of the QDs have a FWHM around $22 \mathrm{~nm}$ at room temperature [from figure 1(d)], the linewidth seems mostly due to in-plane fluctuations of the In content in the QDs and the QD diameter.

The correlation of $\mu \mathrm{PL}$ in-situ APT and of the structural parameters issued by TEM has also been performed at the level of a second tip as described in figure S8 of the Supporting Information, and confirming the reproducibility of the results and the homogeneity of the sample.

In summary, the excellent agreement between the theoretical calculations and the experimental optical features show that the model extracted from structural and chemical characterization provides a good description of this InGaN/GaN QD system. This model conveys an image of the InGaN/GaN QDs that is very different from the Stranski-Krastanov growth in the InGaAs/GaAs or GaN/AIN systems, where the dots and wetting layer can be distinctly and separately visualized. On the contrary, in the structure under study, the thickness of the wetting layer is thicker than the part of the QDs that protrudes above the layer. The dots look resemble rather the coexistence of a thickness undulation and a local increase of the In mole fraction. This perturbed Stranski-Krastanov growth is caused by the

presence of indium at the growth front, with a surfactant effect that favors planar growth. ${ }^{32,61}$ However, these nanostructures behave optically as quantum dots, with an enhanced stability of the luminescence at high temperature thanks to their capability to confine free carriers a three-dimensional volume with a size comparable that of the exciton in InGaN.

\section{CONCLUSIONS AND PERSPECTIVES}

To conclude, we have performed a correlative microscopy study of a stack of self-assembled InGaN/GaN $(2 \mathrm{~nm} / 10 \mathrm{~nm})$ QDs embedded in a needle-shaped atom probe specimen 
fabricated by FIB. Comparative optical characterization of the as-grown sample and the atom probe specimen shows that there was no loss or rearrangement of indium during the FIB process. This allows the extrapolation of the characterization results to the as-grown sample. To get a complete view of the QDs, we have performed spectroscopic (in-situ mocro-photoluminescence and ex-situ cathodoluminescence) and structural (XRD, AFM, STEM and APT) measurements, to get a full view of the sample at the nanometer scale. During APT measurement of the 9 QD layers closer to the substrate, it was possible to resolve single QD emission lines. The spectral red shift observed in these single quantum dot lines during the APT process was assigned to the relaxation of elastic strain due to the material evaporation. Theoretical modeling of the QDs considering the experimental values of their size and indium distribution predicts room temperature photoluminescence at 426 $\mathrm{nm}$, in excellent agreement with the measured spectra. Furthermore, the spectral linewidth of the as-grown sample is explained as due to in-plane fluctuations of the QD In content and diameter. These results confirm the relevance of PAP studies to provide a complete view of the InGaN QDs, and shows the potential of this technique for the analysis of nanostructured materials.

In terms of nanomaterials, a conclusion of this study is that Stranski-Krastranov InGaN/GaN QDs look like a thickness undulation and a local increase of the In mole fraction. The thickness of the wetting layer is comparable to the QD height, and the indium content in the wetting layer is significantly lower than the indium content in the QDs. The nanostructures behave optically as quantum dots, but these structural parameters can have an important impact on the design of the active region of a light emitting device, since they affect the band diagram and carrier transport properties.

In particular, we have shown that PAP can be used in samples containing a large set of nanoscale emitters in order to isolate the optical emission of few of them by a controlled 
reduction of the optically active volume induced by field evaporation. Furthermore, the technique allows the correlation of the 3D image of the chemical composition of a few light emitters contained in a nanoscale object with their spectral signature. This opens perspectives for the application of this technique to super-resolution studies, to discriminate the optical emission of neighboring quantum dots, or to strictly correlative studies, in which the 3D chemical map of a single quantum dot is correlated with its optical signature.

\section{- ASSOCIATED CONTENT}

*Supporting Information: The supporting information includes AFM measurements of the as-grown sample, HAADF and BF STEM images of the InGaN/GaN QD superlattice, crosssection CL mapping of a cleaved edge of the as-grown sample, study of the variation of the CL spectrum along the APT specimen, the $\mu \mathrm{PL}$ spectra recorded in situ during the La-APT characterization of InGaN/GaN QDs, and a calculation of the distribution of the in-plane $\left(\varepsilon_{\mathrm{xx}}\right)$ and out-of-plane $\left(\varepsilon_{\mathrm{zz}}\right)$ strain in the InGaN QD stack.

\section{- ACKNOWLEDGEMENTS}

This work was co-funded in the framework of RIN IFROST, and CPER BRIDGE projects by European Union with European Regional Development Fund (ERDF) and by Region Normandie. This study was performed on the NanoCharacterisation PlatForm (PFNC), and supported by the "Recherches Technologiques de Base" Program of the French Ministry of Research.

\section{- REFERENCES}

(1) Vurgaftman, I.; Meyer, J. R. Band Parameters for Nitrogen-Containing Semiconductors. J. Appl. Phys. 2003, 94 (6), 3675. https://doi.org/10.1063/1.1600519.

(2) Gorczyca, I.; Suski, T.; Christensen, N. E.; Svane, A. Size Effects in Band Gap Bowing in Nitride Semiconducting Alloys. Phys. Rev. B 2011, 83 (15), 153301. 
https://doi.org/10.1103/PhysRevB.83.153301.

(3) Tchernycheva, M.; Nevou, L.; Doyennette, L.; Julien, F.; Warde, E.; Guillot, F.; Monroy, E.; Bellet-Amalric, E.; Remmele, T.; Albrecht, M. Systematic Experimental and Theoretical Investigation of Intersubband Absorption in GaNAlN Quantum Wells. Phys. Rev. B 2006, 73 (12), 125347. https://doi.org/10.1103/PhysRevB.73.125347.

(4) King, P. D. C.; Veal, T. D.; Kendrick, C. E.; Bailey, L. R.; Durbin, S. M.; McConville, C. F. InN/GaN Valence Band Offset: High-Resolution x-Ray Photoemission Spectroscopy Measurements. Phys. Rev. B 2008, 78 (3), 033308. https://doi.org/10.1103/PhysRevB.78.033308.

(5) Nakamura, S.; Pearton, S.; Fasol, G. The Blue Laser Diode: The Complete Story; Springer Berlin Heidelberg: Berlin, Heidelberg, 2000.

(6) Pimputkar, S.; Speck, J. S.; DenBaars, S. P.; Nakamura, S. Prospects for LED Lighting. Nat. Photonics 2009, 3 (4), 180-182. https://doi.org/10.1038/nphoton.2009.32.

(7) Deshpande, S.; Heo, J.; Das, A.; Bhattacharya, P. Electrically Driven Polarized Single-Photon Emission from an InGaN Quantum Dot in a GaN Nanowire. Nat. Commun. 2013, 4 (1), 1675. https://doi.org/10.1038/ncomms2691.

(8) Cho, J.-H.; Kim, Y. M.; Lim, S.-H.; Yeo, H.-S.; Kim, S.; Gong, S.-H.; Cho, Y.-H. Strongly Coherent Single-Photon Emission from Site-Controlled InGaN Quantum Dots Embedded in GaN Nanopyramids. ACS Photonics 2018, 5 (2), 439-444. https://doi.org/10.1021/acsphotonics.7b00922.

(9) Chichibu, S.; Azuhata, T.; Sota, T.; Nakamura, S. Spontaneous Emission of Localized Excitons in InGaN Single and Multiquantum Well Structures. Appl. Phys. Lett. 1996, 69 (27), 4188-4190. https://doi.org/10.1063/1.116981.

(10) Chichibu, S. F.; Abare, A. C.; Minsky, M. S.; Keller, S.; Fleischer, S. B.; Bowers, J. E.; Hu, E.; Mishra, U. K.; Coldren, L. A.; DenBaars, S. P.; Sota, T. Effective Band Gap Inhomogeneity and Piezoelectric Field in InGaN/GaN Multiquantum Well Structures. Appl. Phys. Lett. 1998, 73 (14), 2006-2008. https://doi.org/10.1063/1.122350.

(11) Mancini, L.; Hernández-Maldonado, D.; Lefebvre, W.; Houard, J.; Blum, I.; Vurpillot, F.; Eymery, J.; Durand, C.; Tchernycheva, M.; Rigutti, L. Multi-Microscopy Study of the Influence of Stacking Faults and Three-Dimensional In Distribution on the Optical Properties of m-Plane InGaN Quantum Wells Grown on Microwire Sidewalls. Appl. Phys. Lett. 2016, 108, 04102. https://doi.org/10.1063/1.4940748.

(12) Yang, T.-J.; Shivaraman, R.; Speck, J. S.; Wu, Y.-R. The Influence of Random Indium Alloy Fluctuations in Indium Gallium Nitride Quantum Wells on the Device Behavior. J. Appl. Phys. 2014, 116 (11), 113104. https://doi.org/10.1063/1.4896103.

(13) Vaxenburg, R.; Rodina, A.; Lifshitz, E.; Efros, A. L. The Role of Polarization Fields in Auger-Induced Efficiency Droop in Nitride-Based Light-Emitting Diodes. Appl. Phys. Lett. 2013, 103 (22), 221111. https://doi.org/10.1063/1.4833915.

(14) Iveland, J.; Martinelli, L.; Peretti, J.; Speck, J. S.; Weisbuch, C. Direct Measurement of Auger Electrons Emitted from a Semiconductor Light-Emitting Diode under Electrical Injection: Identification of the Dominant Mechanism for Efficiency Droop. Phys. Rev. Lett. 2013, 110 (17), 177406. https://doi.org/10.1103/PhysRevLett.110.177406.

(15) Moriwaki, O.; Someya, T.; Tachibana, K.; Ishida, S.; Arakawa, Y. Narrow Photoluminescence Peaks from Localized States in InGaN Quantum Dot Structures. Appl. Phys. Lett. 2000, 76 (17), 2361-2363. https://doi.org/10.1063/1.126346.

(16) Park, I.-K.; Kwon, M.-K.; Cho, C.-Y.; Kim, J.-Y.; Cho, C.-H.; Park, S.-J. Effect of InGaN Quantum Dot Size on the Recombination Process in Light-Emitting Diodes. Appl. Phys. Lett. 2008, 92 (25), 253105. https://doi.org/10.1063/1.2951607.

(17) Rigutti, L.; Blum, I.; Shinde, D.; Hernández-Maldonado, D.; Lefebvre, W.; Houard, J.; Vurpillot, F.; Vella, A.; Tchernycheva, M.; Durand, C.; Eymery, J.; Deconihout, B. 
Correlation of Microphotoluminescence Spectroscopy, Scanning Transmission Electron Microscopy, and Atom Probe Tomography on a Single Nano-Object Containing an InGaN/GaN Multiquantum Well System. Nano Lett. 2014, 14 (1), 107-114. https://doi.org/10.1021/n14034768.

(18) Mancini, L.; Moyon, F.; Hernàndez-Maldonado, D.; Blum, I.; Houard, J.; Lefebvre, W.; Vurpillot, F.; Das, A.; Monroy, E.; Rigutti, L. Carrier Localization in GaN/AlN Quantum Dots As Revealed by Three-Dimensional Multimicroscopy. Nano Lett. 2017, 17 (7), 42614269. https://doi.org/10.1021/acs.nanolett.7b01189.

(19) Chamard, V.; Schülli, T.; Sztucki, M.; Metzger, T.; Sarigiannidou, E.; Rouvière, J.-L.; Tolan, M.; Adelmann, C. Strain Distribution in Nitride Quantum Dot Multilayers. Phys. Rev. B 2004, 69 (12), 125327. https://doi.org/10.1103/PhysRevB.69.125327.

(20) Damilano, B.; Grandjean, N.; Dalmasso, S.; Massies, J. Room-Temperature BlueGreen Emission from InGaN/GaN Quantum Dots Made by Strain-Induced Islanding Growth. Appl. Phys. Lett. 1999, 75 (24), 3751-3753. https://doi.org/10.1063/1.125444.

(21) Das, A.; Sinha, P.; Kotsar, Y.; Kandaswamy, P. K.; Dimitrakopulos, G. P.; Kehagias, Th.; Komninou, Ph.; Nataf, G.; De Mierry, P.; Monroy, E. Growth and Characterization of Polar (0001) and Semipolar (11-22) InGaN/GaN Quantum Dots. J. Cryst. Growth 2011, 323 (1), 161-163. https://doi.org/10.1016/j.jcrysgro.2010.10.130.

(22) Bartel, T.; Dworzak, M.; Strassburg, M.; Hoffmann, A.; Strittmatter, A.; Bimberg, D. Recombination Dynamics of Localized Excitons in InGaN Quantum Dots. Appl. Phys. Lett. 2004, 85 (11), 1946-1948. https://doi.org/10.1063/1.1790599.

(23) Park, I.-K.; Kwon, M.-K.; Seo, S.-B.; Kim, J.-Y.; Lim, J.-H.; Park, S.-J. Ultraviolet Light-Emitting Diodes with Self-Assembled InGaN Quantum Dots. Appl. Phys. Lett. 2007, 90 (11), 111116. https://doi.org/10.1063/1.2712804.

(24) Smeeton, T. M.; Kappers, M. J.; Barnard, J. S.; Vickers, M. E.; Humphreys, C. J. Electron-Beam-Induced Strain within InGaN Quantum Wells: False Indium "Cluster" Detection in the Transmission Electron Microscope. Appl. Phys. Lett. 2003, 83 (26), 54195421. https://doi.org/10.1063/1.1636534.

(25) Galtrey, M. J.; Oliver, R. A.; Kappers, M. J.; McAleese, C.; Zhu, D.; Humphreys, C. J.; Clifton, P. H.; Larson, D.; Cerezo, A. Compositional Inhomogeneity of a High-Efficiency InxGa1-xN Based Multiple Quantum Well Ultraviolet Emitter Studied by Three Dimensional Atom Probe. Appl. Phys. Lett. 2008, 92 (4), 041904. https://doi.org/10.1063/1.2829592.

(26) Cadel, E.; Vurpillot, F.; Lardé, R.; Duguay, S.; Deconihout, B. Depth Resolution Function of the Laser Assisted Tomographic Atom Probe in the Investigation of Semiconductors. J. Appl. Phys. 2009, 106 (4), 044908. https://doi.org/10.1063/1.3186617.

(27) Houard, J.; Normand, A.; Di Russo, E.; Bacchi, C.; Dalapati, P.; Beainy, G.; Moldovan, S.; Da Costa, G.; Delaroche, F.; Vaudolon, C.; Chauveau, J. M.; Hugues, M.; Blavette, D.; Deconihout, B.; Vella, A.; Vurpillot, F.; Rigutti, L. A Photonic Atom Probe Coupling 3D Atomic Scale Analysis with in Situ Photoluminescence Spectroscopy. Rev. Sci. Instrum. 2020, 91 (8), 083704. https://doi.org/10.1063/5.0012359.

(28) Mancini, L.; Moyon, F.; Houard, J.; Blum, I.; Lefebvre, W.; Vurpillot, F.; Das, A.; Monroy, E.; Rigutti, L. Multi-Excitonic Emission from Stranski-Krastanov GaN/AlN Quantum Dots inside a Nanoscale Tip. Appl. Phys. Lett. 2017, 111 (24), 243102. https://doi.org/10.1063/1.5004417.

(29) Di Russo, E.; Cherkashin, N.; Korytov, M. N.; Nikolaev, A. E.; Sakharov, A. V.; Tsatsulnikov, A. F.; Bonef, B.; Blum, I.; Houard, J.; da Costa, G.; Blavette, D.; Rigutti, L. Compositional Accuracy in Atom Probe Tomography Analyses Performed on III-N Light Emitting Diodes. J. Appl. Phys. 2019, 126 (12), 124307. https://doi.org/10.1063/1.5113799.

(30) Valdueza-Felip, S.; Bellet-Amalric, E.; Núñez-Cascajero, A.; Wang, Y.; Chauvat, M.- 
P.; Ruterana, P.; Pouget, S.; Lorenz, K.; Alves, E.; Monroy, E. High In-Content InGaN Layers Synthesized by Plasma-Assisted Molecular-Beam Epitaxy: Growth Conditions, Strain Relaxation, and In Incorporation Kinetics. J. Appl. Phys. 2014, 116 (23), 233504. https://doi.org/10.1063/1.4903944.

(31) Gačević, Ž.; Das, A.; Teubert, J.; Kotsar, Y.; Kandaswamy, P. K.; Kehagias, Th.; Koukoula, T.; Komninou, Ph.; Monroy, E. Internal Quantum Efficiency of III-Nitride Quantum Dot Superlattices Grown by Plasma-Assisted Molecular-Beam Epitaxy. J. Appl. Phys. 2011, 109 (10), 103501. https://doi.org/10.1063/1.3590151.

(32) Neugebauer, J.; Zywietz, T.; Scheffler, M.; Northrup, J.; Chen, H.; Feenstra, R. Adatom Kinetics On and Below the Surface: The Existence of a New Diffusion Channel. Phys. Rev. Lett. 2003, 90 (5), 056101. https://doi.org/10.1103/PhysRevLett.90.056101.

(33) Bennett, S. E.; Saxey, D. W.; Kappers, M. J.; Barnard, J. S.; Humphreys, C. J.; Smith, G. D.; Oliver, R. A. Atom Probe Tomography Assessment of the Impact of Electron Beam Exposure on In ${ }_{x} \mathrm{Ga}_{1-\mathrm{x}} \mathrm{N} / \mathrm{GaN}$ Quantum Wells. Appl. Phys. Lett. 2011, 99 (2), 021906. https://doi.org/10.1063/1.3610468.

(34) Blum, I.; Cuvilly, F.; Lefebvre-Ulrikson, W. Atom Probe Sample Preparation. In Atom Probe Tomography; Elsevier, 2016; pp 97-121. https://doi.org/10.1016/B978-0-12804647-0.00004-8.

(35) Rigutti, L.; Venturi, L.; Houard, J.; Normand, A.; Silaeva, E. P.; Borz, M.; Malykhin, S. A.; Obraztsov, A. N.; Vella, A. Optical Contactless Measurement of Electric Field-Induced Tensile Stress in Diamond Nanoscale Needles. Nano Lett. 2017, 17 (12), 7401-7409. https://doi.org/10.1021/acs.nanolett.7b03222.

(36) Birner, S.; Zibold, T.; Andlauer, T.; Kubis, T.; Sabathil, M.; Trellakis, A.; Vogl, P. Nextnano: General Purpose 3-D Simulations. IEEE Trans. Electron Devices 2007, 54 (9), 2137-2142. https://doi.org/10.1109/TED.2007.902871.

(37) Suski, T.; Staszczak, G.; Korona, K. P.; Lefebvre, P.; Monroy, E.; Drozdz, P. A.; Muzioł, G.; Skierbiszewski, C.; Kulczykowski, M.; Matuszewski, M.; Grzanka, E.; Grzanka, S.; Pieniak, K.; Gibasiewicz, K.; Khachapuridze, A.; Smalc-Koziorowska, J.; Marona, L.; Perlin, P. Switching of Exciton Character in Double InGaN/GaN Quantum Wells. Phys. Rev. B 2018, 98 (16), 165302. https://doi.org/10.1103/PhysRevB.98.165302.

(38) Tchernycheva, M.; Nevou, L.; Doyennette, L.; Julien, F.; Warde, E.; Guillot, F.; Monroy, E.; Bellet-Amalric, E.; Remmele, T.; Albrecht, M. Systematic Experimental and Theoretical Investigation of Intersubband Absorption in GaNAIN Quantum Wells. Phys. Rev. B 2006, 73 (12), 125347. https://doi.org/10.1103/PhysRevB.73.125347.

(39) Kandaswamy, P. K.; Guillot, F.; Bellet-Amalric, E.; Monroy, E.; Nevou, L.; Tchernycheva, M.; Michon, A.; Julien, F. H.; Baumann, E.; Giorgetta, F. R.; Hofstetter, D.; Remmele, T.; Albrecht, M.; Birner, S.; Dang, L. S. GaN/AlN Short-Period Superlattices for Intersubband Optoelectronics: A Systematic Study of Their Epitaxial Growth, Design, and Performance. J. Appl. Phys. 2008, 104 (9), 093501. https://doi.org/10.1063/1.3003507.

(40) Das, A.; Dimitrakopulos, G. P.; Kotsar, Y.; Lotsari, A.; Kehagias, Th.; Komninou, Ph.; Monroy, E. Improved Luminescence and Thermal Stability of Semipolar (11-22) InGaN Quantum Dots. Appl. Phys. Lett. 2011, 98 (20), 201911. https://doi.org/10.1063/1.3588335.

(41) Gogneau, N.; Jalabert, D.; Monroy, E.; Sarigiannidou, E.; Rouvière, J. L.; Shibata, T.; Tanaka, M.; Gerard, J. M.; Daudin, B. Influence of AlN Overgrowth on Structural Properties of GaN Quantum Wells and Quantum Dots Grown by Plasma-Assisted Molecular Beam Epitaxy. J. Appl. Phys. 2004, 96 (2), 1104-1110. https://doi.org/10.1063/1.1759785.

(42) Figge, S.; Tessarek, C.; Aschenbrenner, T.; Hommel, D. InGaN Quantum Dot Growth in the Limits of Stranski-Krastanov and Spinodal Decomposition: InGaN Quantum Dots. Phys. Status Solidi B 2011, 248 (8), 1765-1776. https://doi.org/10.1002/pssb.201147165.

(43) Lefebvre, P.; Taliercio, T.; Morel, A.; Allègre, J.; Gallart, M.; Gil, B.; Mathieu, H.; 
Damilano, B.; Grandjean, N.; Massies, J. Effects of GaAlN Barriers and of Dimensionality on Optical Recombination Processes in InGaN Quantum Wells and Quantum Boxes. Appl. Phys. Lett. 2001, 78 (11), 1538-1540. https://doi.org/10.1063/1.1352664.

(44) Adelmann, C.; Simon, J.; Feuillet, G.; Pelekanos, N. T.; Daudin, B.; Fishman, G. Self-Assembled InGaN Quantum Dots Grown by Molecular-Beam Epitaxy. Appl. Phys. Lett. 2000, 76 (12), 1570-1572. https://doi.org/10.1063/1.126098.

(45) Zhu, T.; El-Ella, H. A. R.; Reid, B.; Holmes, M. J.; Taylor, R. A.; Kappers, M. J.; Oliver, R. A. Growth and Optical Characterisation of Multilayers of InGaN Quantum Dots. J. Cryst. Growth 2012, 338 (1), 262-266. https://doi.org/10.1016/j.jcrysgro.2011.11.001.

(46) Polian, A.; Grimsditch, M.; Grzegory, I. Elastic Constants of Gallium Nitride. J. Appl. Phys. 1996, 79 (6), 3343-3344. https://doi.org/10.1063/1.361236.

(47) Wright, A. F. Elastic Properties of Zinc-Blende and Wurtzite AlN, GaN, and InN. $J$. Appl. Phys. 1997, 82 (6), 2833-2839. https://doi.org/10.1063/1.366114.

(48) Andreev, A.; O'Reilly, E. Theory of the Electronic Structure of GaN/AlN Hexagonal Quantum Dots. Phys. Rev. B 2000, 62 (23), 15851-15870. https://doi.org/10.1103/PhysRevB.62.15851.

(49) Sarigiannidou, E.; Monroy, E.; Daudin, B.; Rouvière, J. L.; Andreev, A. D. Strain Distribution in GaNAIN Quantum-Dot Superlattices. Appl. Phys. Lett. 2005, 87 (20), 203112. https://doi.org/10.1063/1.2123394.

(50) Himwas, C.; den Hertog, M.; Bellet-Amalric, E.; Songmuang, R.; Donatini, F.; Si Dang, L.; Monroy, E. Enhanced Room-Temperature Mid-Ultraviolet Emission from AlGaN/AlN Stranski-Krastanov Quantum Dots. J. Appl. Phys. 2014, 116 (2), 023502. https://doi.org/10.1063/1.4887140.

(51) Leroux, M.; Grandjean, N.; Beaumont, B.; Nataf, G.; Semond, F.; Massies, J.; Gibart, P. Temperature Quenching of Photoluminescence Intensities in Undoped and Doped GaN. $J$. Appl. Phys. 1999, 86 (7), 3721-3728. https://doi.org/10.1063/1.371242.

(52) Rubanov, S.; Munroe, P. R. FIB-Induced Damage in Silicon. J. Microsc. 2004, 214 (3), 213-221. https://doi.org/10.1111/j.0022-2720.2004.01327.x.

(53) Bogdanowicz, J.; Kumar, A.; Fleischmann, C.; Gilbert, M.; Houard, J.; Vella, A.; Vandervorst, W. Laser-Assisted Atom Probe Tomography of Semiconductors: The Impact of the Focused-Ion Beam Specimen Preparation. Ultramicroscopy 2018, 188, 19-23. https://doi.org/10.1016/j.ultramic.2018.03.001.

(54) Dhara, S.; Datta, A.; Wu, C. T.; Chen, K. H.; Wang, Y. L.; Muto, S.; Tanabe, T.; Shen, C. H.; Hsu, C. W.; Chen, L. C.; Maruyama, T. Mechanism of Nanoblister Formation in Ga+ Self-Ion Implanted GaN Nanowires. Appl. Phys. Lett. 2005, 86 (20), 203119. https://doi.org/10.1063/1.1931819.

(55) Wu, S.-E.; Hsueh, T.-H.; Liu, C.-P.; Sheu, J.-K.; Lai, W.-C.; Chang, S.-J. Focused Ion Beam Milled InGaN/GaN Multiple Quantum Well Nanopillars. Jpn. J. Appl. Phys. 2008, 47 (4), 3130-3133. https://doi.org/10.1143/JJAP.47.3130.

(56) Rigutti, L. Laser-Assisted Atom Probe Tomography. In Advances in Imaging and Electron Physics; Elsevier, 2020; Vol. 213, pp 29-53. https://doi.org/10.1016/bs.aiep.2019.11.003.

(57) Vurgaftman, I.; Meyer, J. R. Band Parameters for Nitrogen-Containing Semiconductors. J. Appl. Phys. 2003, 94 (6), 3675. https://doi.org/10.1063/1.1600519.

(58) Wei, W.; Jiang, K.; Wei, Y.; Liu, M.; Yang, H.; Zhang, L.; Li, Q.; Liu, L.; Fan, S. Measuring the Stress in Field-Emitting Carbon Nanotubes. Nanotechnology 2006, 17 (8), 1994-1998. https://doi.org/10.1088/0957-4484/17/8/033.

(59) Costa, G. D.; Wang, H.; Duguay, S.; Bostel, A.; Blavette, D.; Deconihout, B. Advance in Multi-Hit Detection and Quantization in Atom Probe Tomography. Rev. Sci. Instrum. 2012, 83 (12), 123709. https://doi.org/10.1063/1.4770120. 
(60) Rigutti, L.; Vella, A.; Vurpillot, F.; Gaillard, A.; Sevelin-Radiguet, N.; Houard, J.; Hideur, A.; Martel, G.; Jacopin, G.; Luna Bugallo, A. D.; Deconihout, B. Coupling Atom Probe Tomography and Photoluminescence Spectroscopy: Exploratory Results and $\begin{array}{llll}\text { Perspectives. } & \text { Ultramicroscopy } & \text { 2013, } & \text { 75-80. }\end{array}$ https://doi.org/10.1016/j.ultramic.2013.02.002.

(61) Chen, H.; Feenstra, R. M.; Northrup, J. E.; Zywietz, T.; Neugebauer, J.; Greve, D. W. Surface Structures and Growth Kinetics of InGaN(0001) Grown by Molecular Beam Epitaxy. J. Vac. Sci. Technol. B Microelectron. Nanometer Struct. 2000, 18 (4), 2284. https://doi.org/10.1116/1.1306296. 


\section{Figure 1}
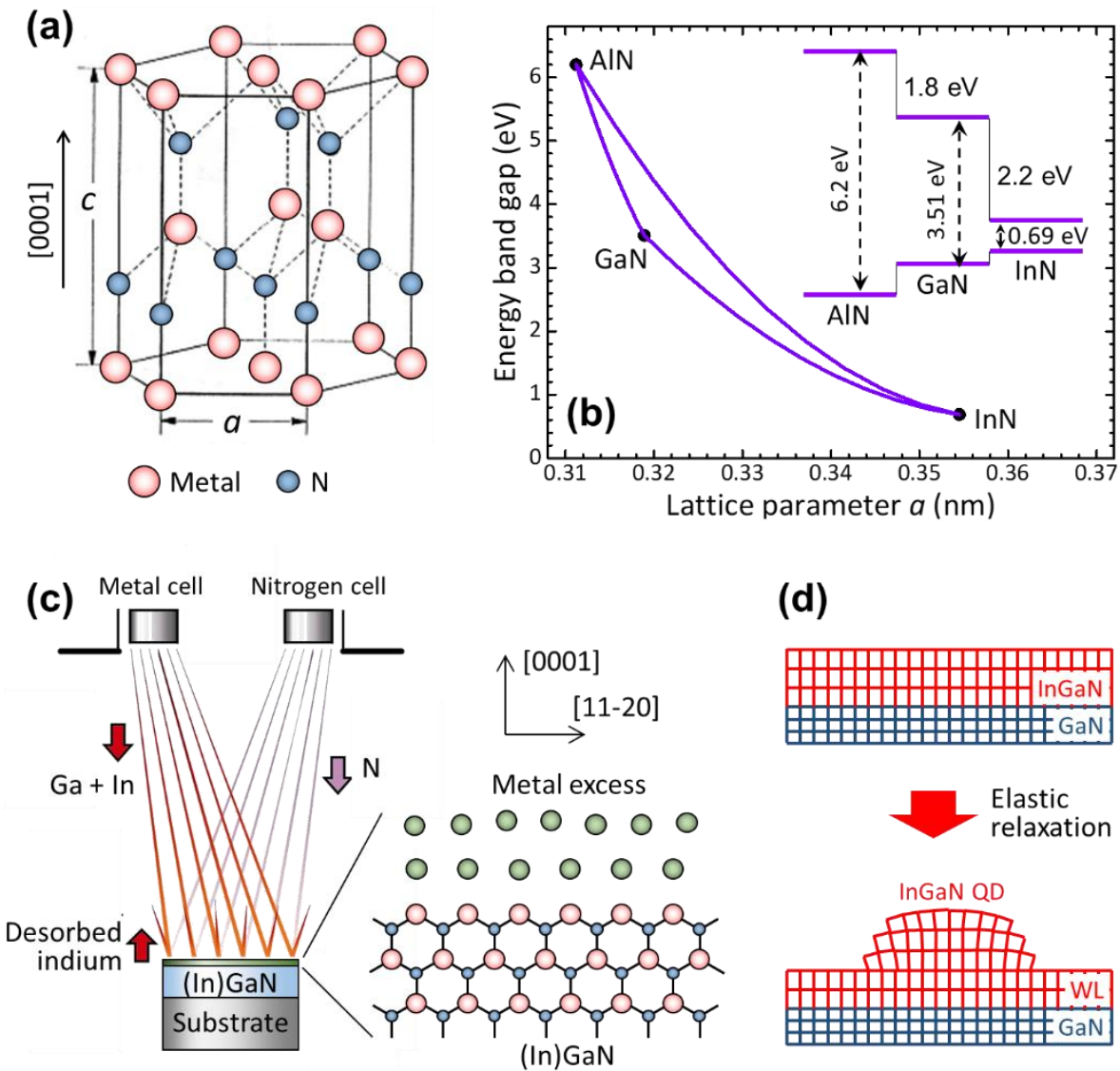

(d)
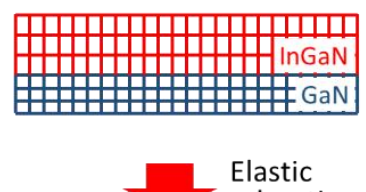

relaxation

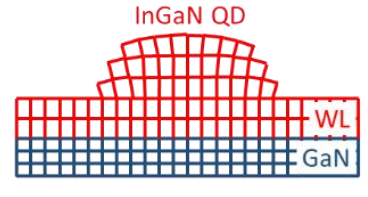

Figure 1. (a) Crystallographic structure of wurtzite III-nitrides. The [0001] growth axis and the inplane (a) and out of plane (c) lattice parameters are indicated in the figure. (b) Relationship between the energy band gap and the in-plane lattice parameter in bulk wurtzite III-nitrides (AlN, GaN, InN and their ternary compounds). Inset: Low temperature band offsets in the AlN / GaN / InN system. 3,4 (c) Schematic description of the PAMBE growth process. Atomic flows of $\mathrm{Ga}$, In and $\mathrm{N}$ imping the substrate surface. At the QD growth temperature, only indium is desorbed. The crystal grows in a metal rich environment, as described in the right side of the image. (d) Formation of InGaN QDs on $\mathrm{GaN}$. The strong lattice mismatch leads to an elastic relaxation when the $\mathrm{InGaN}$ layer reaches a certain critical thickness. The resulting structure consist of InGaN islands (QDs) that are linked by a wetting layer (WL). 
Figure 2
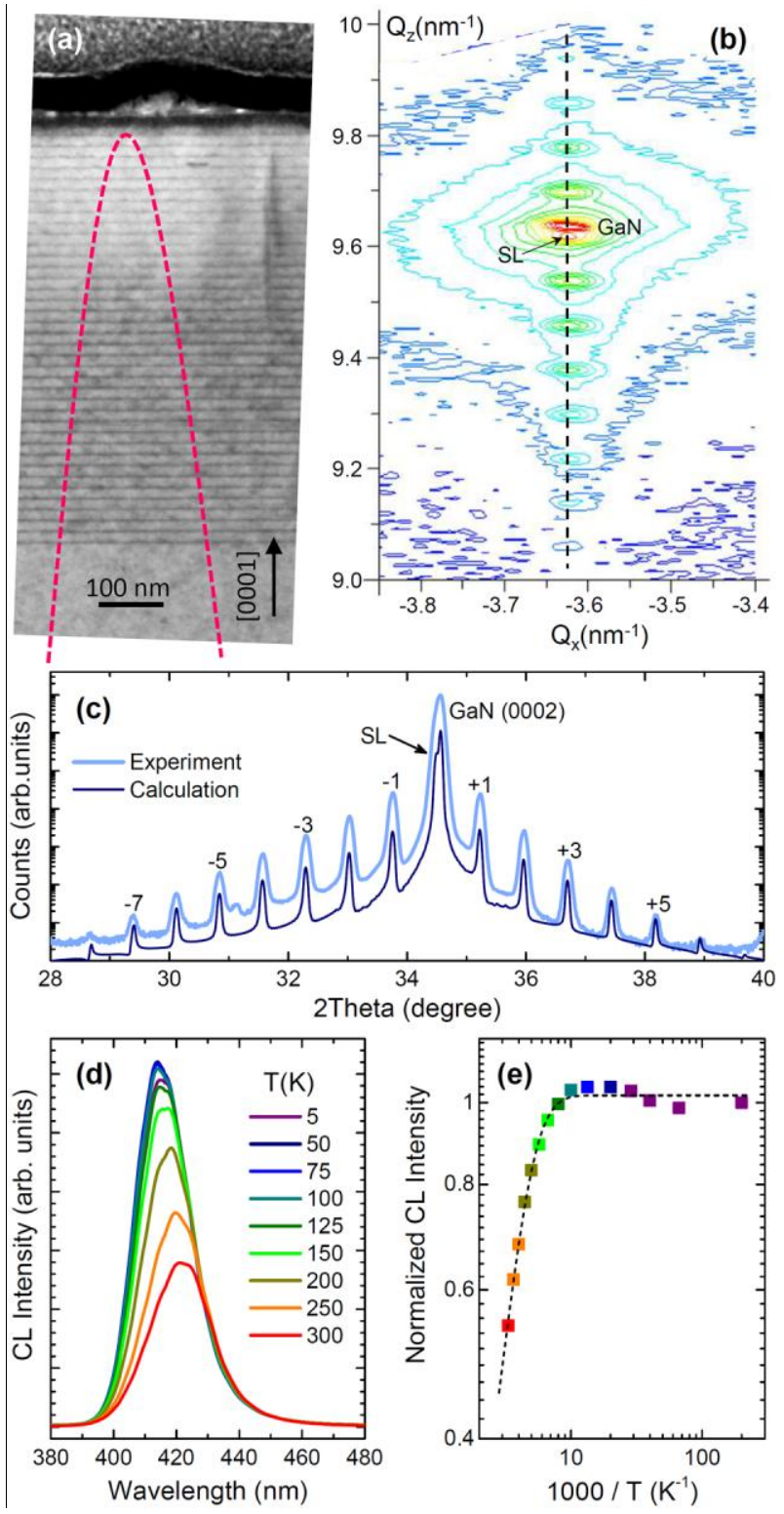

Figure 2. (a) BF-STEM image of the heterostructure showing the 40 periods of InGaN QDs. (b) Reciprocal space map around the (10-15) x-ray reflection of the GaN/InGaN QD superlattice (SL) grown on GaN. The dashed line outlines the shape of the tip specimen that is analyzed atom probe tomography. (c) XRD $\theta-2 \theta$ scan (light blue) recorded around the (0002) reflection of GaN. Labels indicate the (0002) reflection of $\mathrm{GaN}$, and the (0002) reflection of the QD superlattice, with several satellites. The experimental result is compared with a theoretical calculation (dark blue). (d) CL spectra of the as-grown sample recorded at different temperatures. (e) Normalized CL intensity as a function of the inverse temperature. The dashed line is a fit that assumes dominance of a monoexponential nonradiative process. 
Figure 3
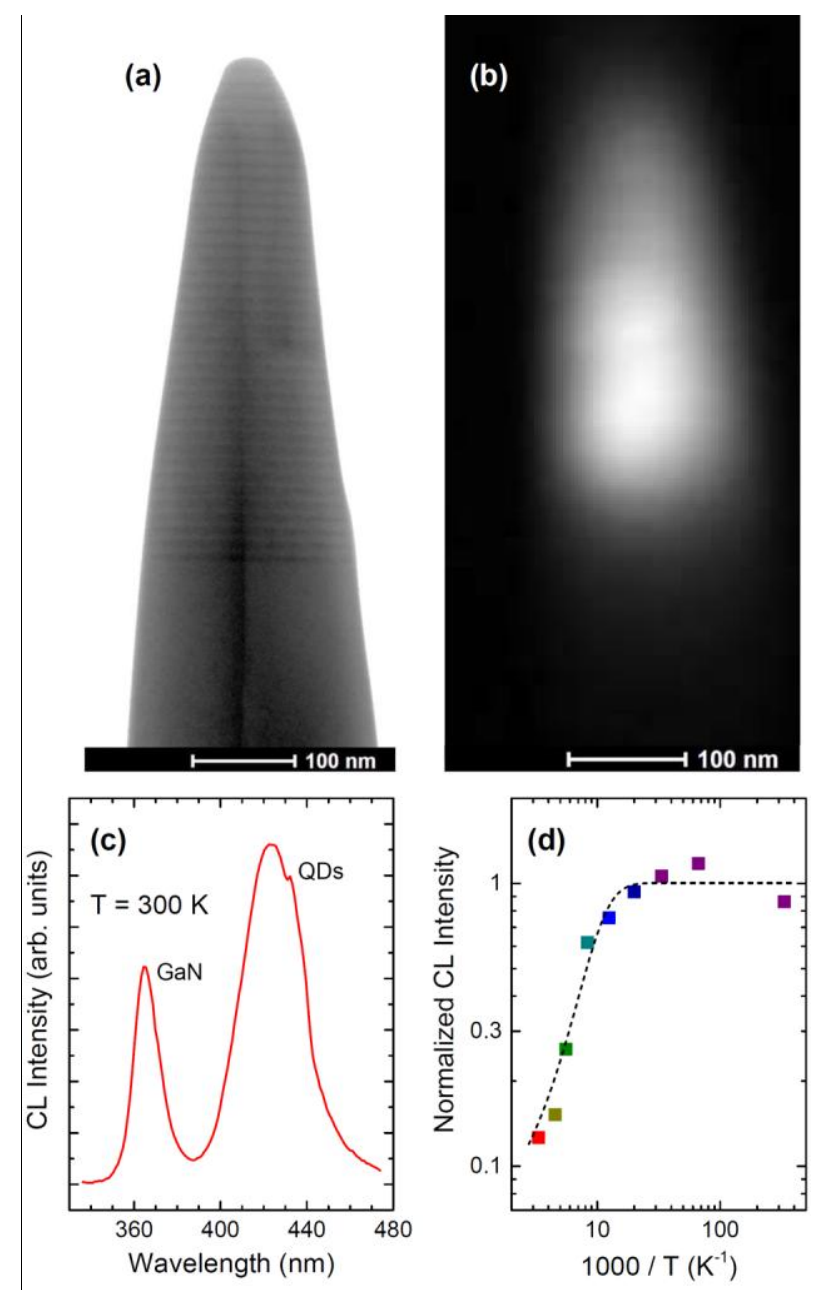

Figure 3. (a) TEM image of the tip after FIB preparation. (b) Panchromatic CL map of the needleshaped specimen in (a). (c) CL spectrum at room temperature, where labels indicate the emission bands associated to the GaN substrate and to the QD superlattice. (d) Normalized CL intensity of the specimen as a function of temperature. The dashed line is a fit that assumes dominance of a monoexponential nonradiative process. 
Figure 4
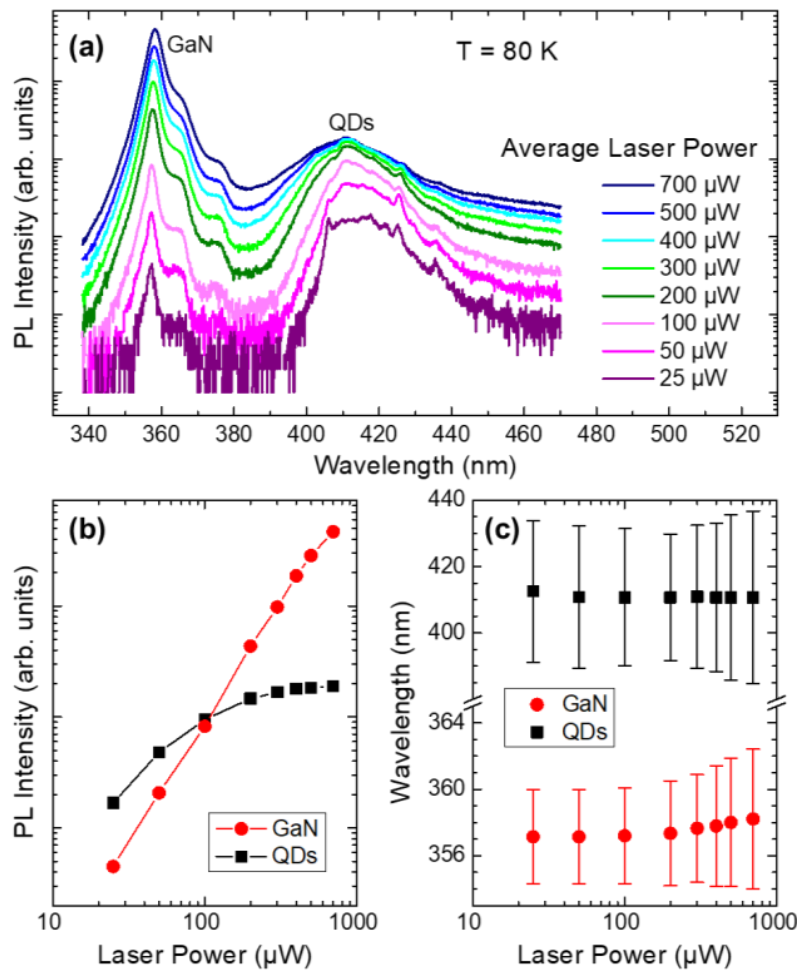

Figure 4. $\mu \mathrm{PL}$ measurements performed before the La-APT evaporation of InGaN/GaN QDs with different laser power. The experiment was performed at $80 \mathrm{~K}$ and the frequency and wavelength of laser were $300 \mathrm{kHz}$ and $260 \mathrm{~nm}$, respectively. (b) Intensity of the GaN and QD PL lines as a function of the laser power, extracted from (a). (c) Peak wavelength of the GaN and QD PL lines as a function of the laser power. The FWHM is indicated as an error bar. 
Figure 5

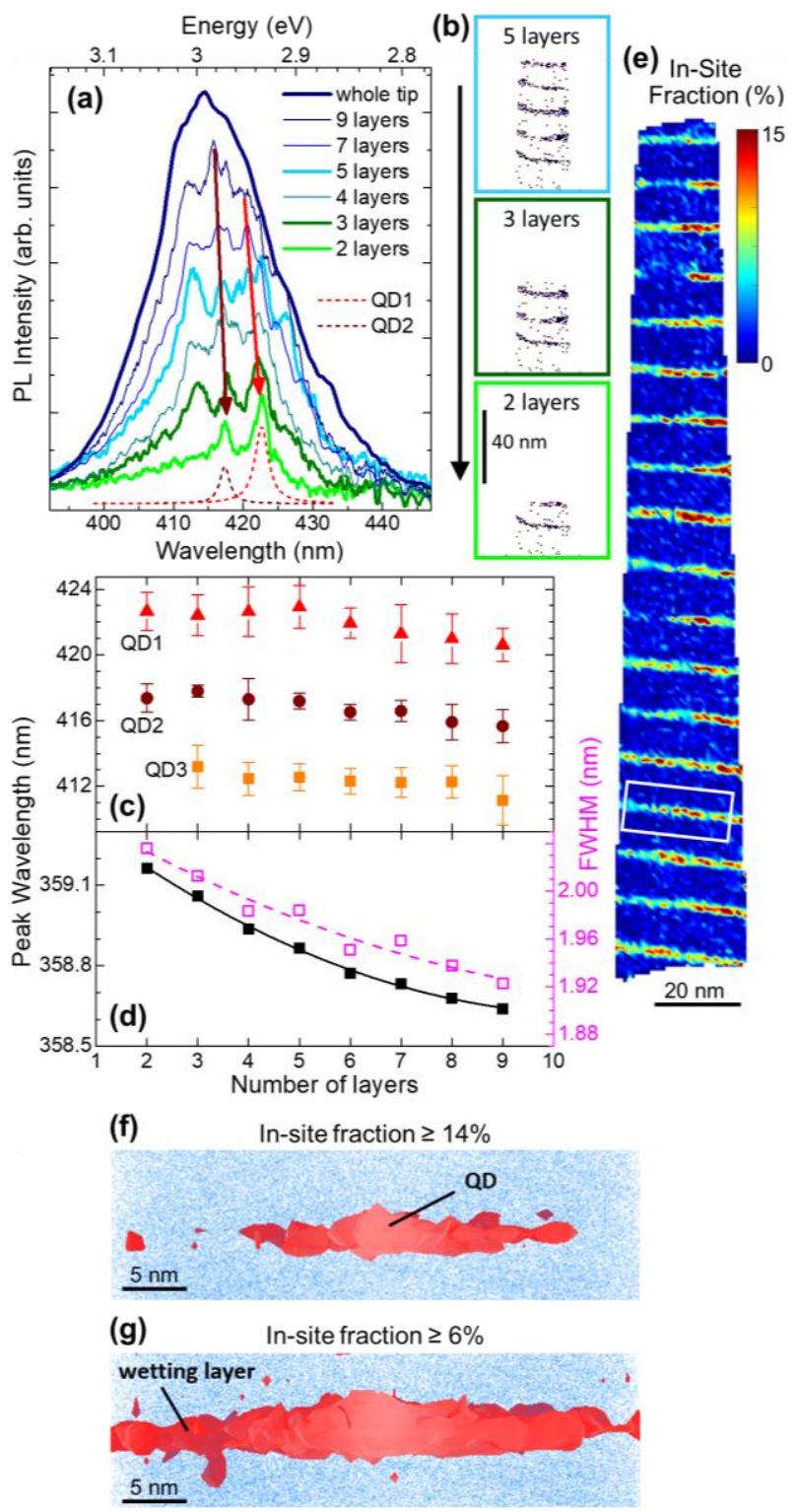

Figure 5. (a) In-situ PL measurements performed during the La-APT evaporation of InGaN/GaN QDs. The experiment was performed at $80 \mathrm{~K}$. The legend indicates the number of layers that remain in the specimen when recording the PL data. Dashed lines are Lorentzian fits that identify the QDs contained in layer 2. Their evolution of the emission of these two QDs is marked with arrows. (b) Indium 3D APT reconstruction of the last 2, 3 and 5 layers of QDs detected during analysis. (c) $\mu \mathrm{PL}$ peak wavelength of three QDs (the signals of QD1 and QD2 disappeared after evaporation of layer 2, as shown in (b), and QD3 had disappeared after evaporation of layer 3). The FWHM is indicated as an error bar. (d) $\mu \mathrm{PL}$ peak wavelength of the $\mathrm{GaN}$ emission vs. number of layers remaining on the specimen during evaporation. The evolution of the FWHM is indicated with open symbols (right axis). (e) Indium-site fraction map calculated from an APT 3D reconstructed volume. In this twodimensional representation, the composition is integrated over $10 \mathrm{~nm}$ in the direction perpendicular to the page. (f-g) In red, isosurfaces enclosing (f) the QD region (indium fraction $\geq 14 \%$ ), and ( $\mathrm{g}$ ) the wetting layer (In fraction $\geq 6 \%$ ), extracted for the layer indicated in figure 5(e). 
Figure 6
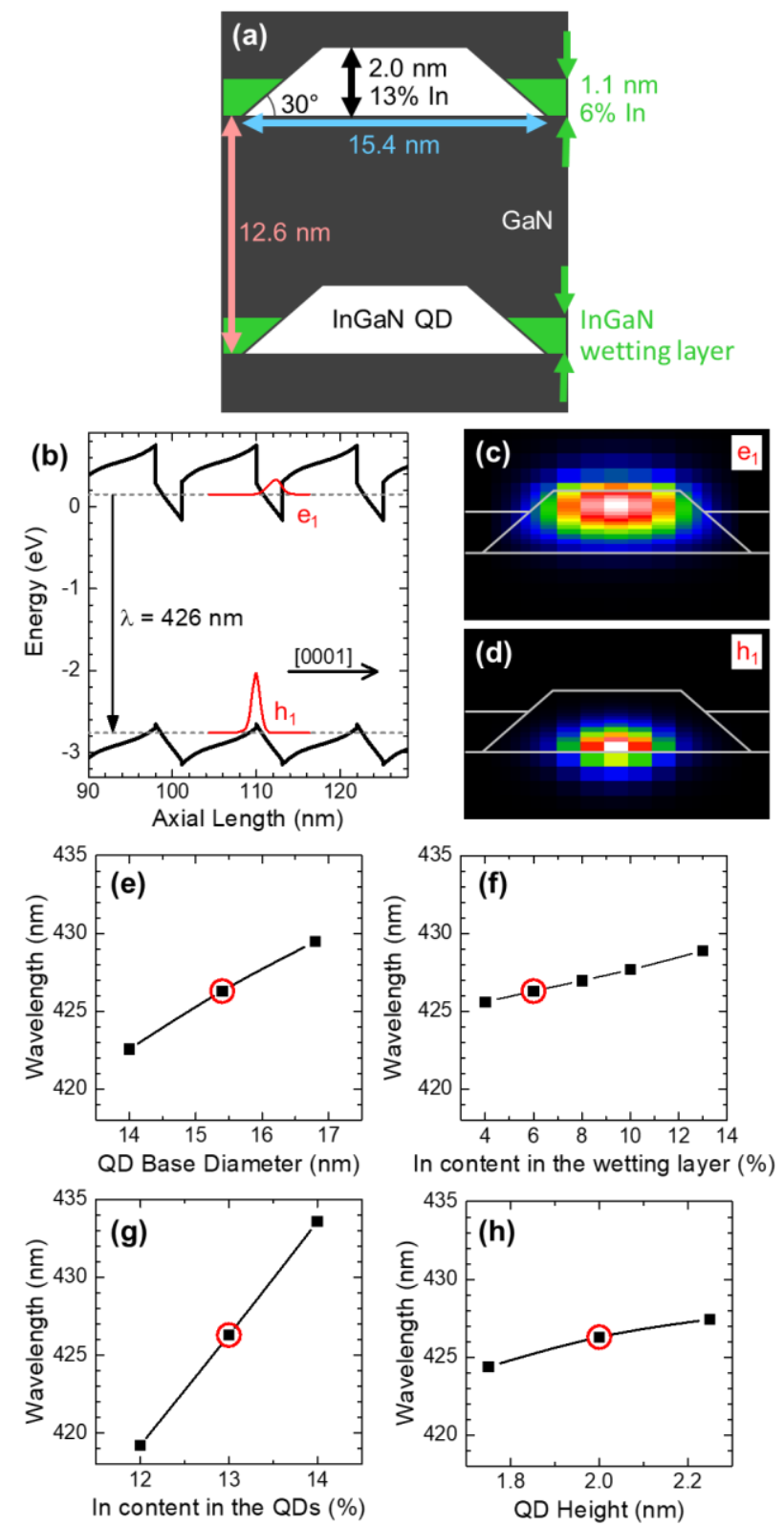

Figure 6. (a) Schematic description of the QDs with composition and dimensions extracted by combination of XRD, AFM, TEM and APT measurements. (b) From 3D Schrödinger-Poisson calculations at room temperature, band profile along [0001] crossing the center of the QDs, with the (c) electron and (d) hole probability distribution obtained by projecting their squared wavefunctions. (e-h) Calculation of the emission wavelength at room temperature as a function of the (e) QD base diameter, (f) indium content in the WL, (g) indium content in the QD, and (h) QD height. In (e-h), red circles outline the result of the simulation with the input parameters described in (a). 
TOC Graphic
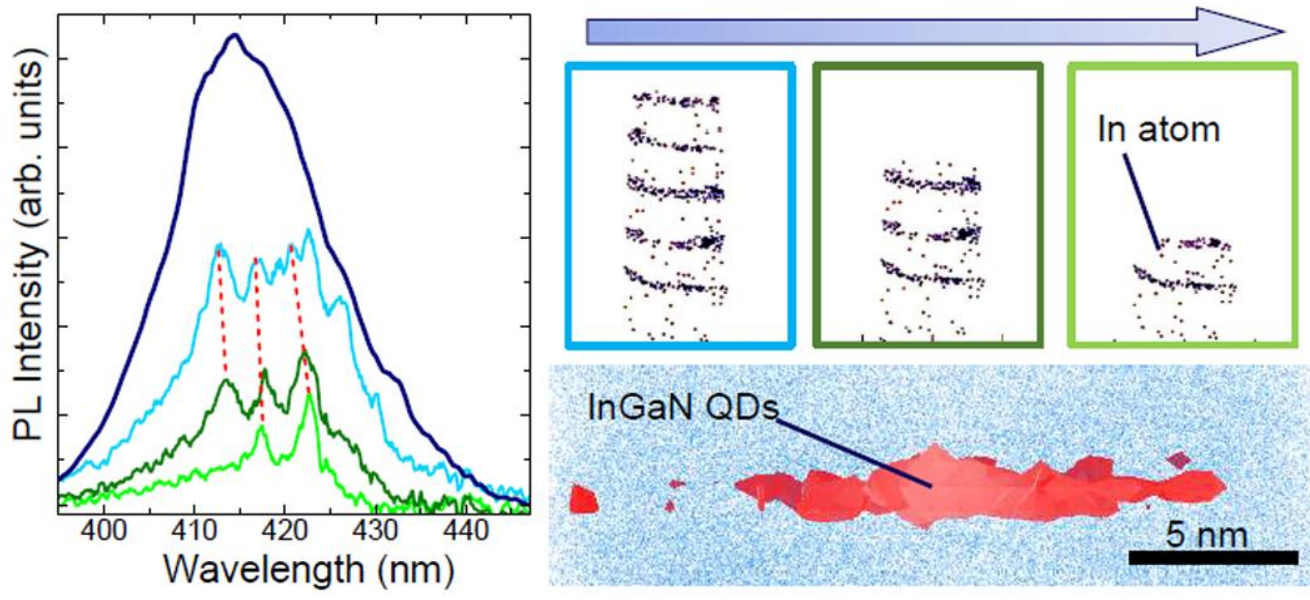